be seriously reconsidered and perhaps abandoned. Possibly, however, complete resection of the thymus cannot be assured whatever operative procedure is used.

${ }^{1}$ Kirschner PA, Osserman KE, Kark AE. Studies in myasthenia gravis. Transcervical total thymectomy. $¥ A M A$ 1969;209:906-10.

2 Papatestas AE, Alpert LI, Osserman KE, Osserman RS, Kark AE. Studies in myasthenia gravis: effects of thymectomy. Am 7 Med 1971;50: 465-74.

${ }^{3}$ Twomey JJ, Lewis VM, Patten BM, Goldstein G, Good RA. Myasthenia gravis, thymectomy and serum thymic hormone activity. $A m \mathcal{f} \mathrm{Med}$ $1979 ; 66: 639-43$.

${ }^{4}$ Rubin M, Straus B, Allen L. Clinical disorders associated with thymic tumors. Arch Intern Med 1964;114:389-98.

${ }^{5}$ Harvey AM. Some preliminary observation on the clinical course of myasthenia gravis before and after thymectomy. Bull NY Acad Med $1948 ; 24: 505-22$.

(Accepted 11 February 1981)

Centro Nacional de Rehabilitación Respiratoria "Maria Ferrer,"

E Finochietto 849, Buenos Aires 1272, Argentina MOISES ROSENBERG, $M D$, chief surgeon AQUILES J RONCORONI, MD, FCCP, medical director

\section{Infective dose of Campylobacter jejuni in milk}

Unpasteurised milk is now recognised as a vehicle of infection for Campylobacter jejuni. Fourteen milk-associated outbreaks involving more than $\mathbf{4 0 0 0}$ cases have been investigated in the last three years in Great Britain, and there is evidence that sections of the population that regularly consume unpasteurised milk have a higher level of detectable antibodies to $C$ jejuni than does the general population (unpublished observations). One of the features of these outbreaks has been the enormous dilution of the original contaminating dose in the bulk tank in which the milk is stored. As $C$ jejuni, unlike salmonellae, will not grow in milk, 1 the occurrence of cases suggests a very low infective dose.

\section{Case report}

On 6 December, two hours after a light breakfast, I swallowed 500 organisms of a known serotype of $C$ jejuni in $180 \mathrm{ml}$ of pasteurised milk. The strain originated from a milk-borne outbreak it: 1970. Faeces were examined twice in the week before the experiment and Lumplohacty was not found Faeces were then examined daily from the start of the experiment and $C$ jejuni of the same serotype was cultured at a titre of at leasi $10^{6} / \mathrm{g}$ from a specimen obtained on day 2 . The count increased to $9 \because 10^{7} / \mathrm{g}$ on day 5 and declined thereafter. Abdominal cramps and mild diarrhowa containing mucus but no blood developed on day 4 and lasted three days. Complement-fixing antibody to $C$ jejuni, which was not present before or on the day of the experiment, was detected at a titro of : te $1 . .1 \%$

\section{Comment}

It is clear that infection can follow the ingestion of small doses of $C$ jejuni when the organism is taken in milk. This finding helps to define the mechanisms concerned in milk-borne outbreaks of $C$ jejuni in man. In addition, although a human experiment has been reported before ${ }^{2} I$ think this is the first time that the requirements of Koch's postulates have been met definitively for $C$ jejuni in man.

1 Blaser MJ, Hardesty HL, Powers B, Wang W-LL. Survival of Campylobacter fetus subsp jejuni in biological milieus. 7 Clin Microbiol 1980;11 : 309-13.

2 Steele TW, McDermott S. Campylobacter enteritis in South Australia Med $\mathcal{7}$ Aust 1978;2:404-6.

(Accepted 16 February 1981)

Public Health Laboratory, Withington Hospital, Manchester M208LR D A ROBINSON, MD, MSC, consultant epidemiologist

\section{Spinal meningioma presenting as focal epilepsy: a case report}

Hughlings Jackson described epilepsy as "occasional, sudden, excessive, rapid, and local discharge of grey matter." By using the term "local" he implied that these attacks could take the form of a focal disorder of function and that anatomical localisation could be deduced from the nature of such an attack. In considering focal motor convul- $\mathbb{D}$ sions, one normally thinks in terms of a cerebral cortical or subcortical seizure source and rarely of a spinal source for such an event, though this is anatomically and physiologically feasible.

\section{Case report}

A 67-year-old right-handed woman was referred initially to this institute in October 1979 with a one-month history of stereotyped involuntary movements affecting her right foot. These movements occurred spontaneously, at rest, or when walking; lasted for several seconds to a minute and occurred many times a day. She had also noticed, before admission, dragging of the right leg. She had sustained a trivial head injury in January 1979 without loss of consciousness, but otherwise there was little of note in her medical history. At the time of her referral she was not taking any medication.

On examination she was obese, but otherwise normal. She suffered from Horner's syndrome in the left eye, which was apparently of long standing, but cranial nerves and arms were otherwise normal. She showed grade 3/5 weakness of right ankle dorsiflexion with mild increase in tone and brisk tendon reflexes on the right with an extensor plantar response. Attacks were observed: these were not precipitated by cutaneous stimulation, but took the form of tonic inversion and plantar flexion of the foot followed by $\partial$ relaxation. There was no change in conscious level throughout. $X$-ray examinations of the skull and cervical spine and a computed tomography scan of the brain (including views of the high motor cortex) showed no abnor- $f$ mality. Haemoglobin concentrations, full blood count, and results of routine biochemical tests were normal. An electroencephalogram showed nonspecific theta activity bilaterally.

The patient was discharged with a diagnosis of focal epilepsy presumed to be of cortical origin. She was started on carbamazepine and subsequently kept under review. Two months later she was seen as an outpatient; her right leg appeared weaker, and the abnormal involuntary movements were occurring up to 20 times a day. There now appeared spinothalamic sensory loss in the contralateral leg with an ill-defined level on the trunk. In view of this the patient was readmitted and myelography performed (figure). This showed an intradural extramedullary lesion at the level of the 11th dorsal vertebra,

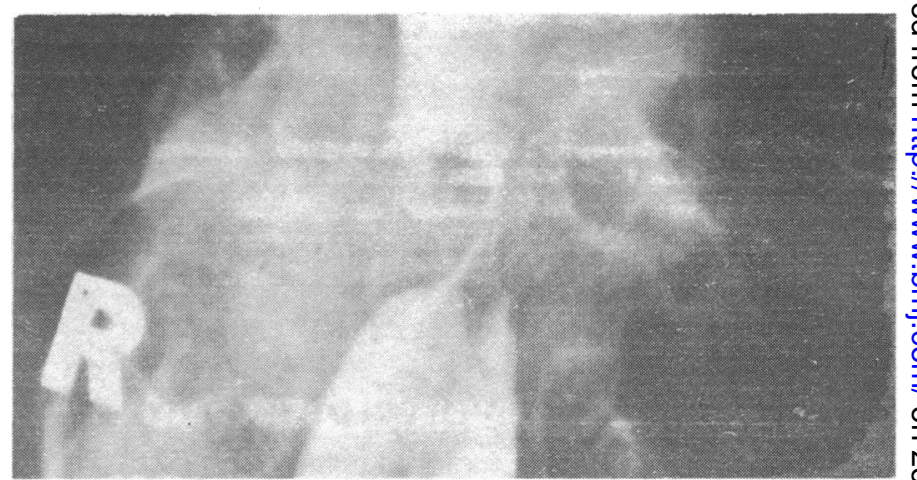

Myelogram showing intradural extramedullary lesion at level of 11 th dorsal vertebra.

anterior and to the right of the spinal cord, which was compressed towards the left. Laminectomy was performed and a right anterolateral meningioma removed. No further focal seizures occurred, and eight months after operation the patient was walking with no discernable weakness, carbamazepine having $\bullet$ been stopped.

\section{Comment}

The dorsal meningioma in this case was in a suitable position to cause damage to the lateral corticospinal tract, and our clinical impression was that these motor events were spinal focal motor seizures

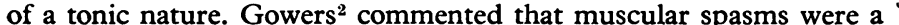
common symptom in spinal tumours and emphasised their diagnostic value. The spasm as he described it was generally preceded by pain and when persistent would often produce contractures. Reflex spasms occur usually in response to sensory stimulation. The movements 